\title{
Propiedades psicométricas de las Escalas de Atribuciones sobre las Causas de la Pobreza y Actitudes hacia los Pobres
}

\author{
Psychometric properties of the Attributions About the Causes \\ of Poverty Scale and the Attitudes Toward the Poor Scale
}

\author{
Cecilia Reyna ${ }^{1}$ \\ Universidad Nacional de Córdoba, CONICET, Argentina
}

\author{
María Florencia Reparaz ${ }^{2}$ \\ Universidad de la Cuenca del Plata, Argentina
}

\begin{abstract}
Resumen. El objetivo del estudio fue analizar las propiedades psicométricas de la Escala de Atribuciones sobre las Causas de la Pobreza y la dimensión afectiva de la Escala de Actitudes hacia los Pobres en el contexto local. La muestra estuvo compuesta por 177 estudiantes universitarios que asistían a dos instituciones de Corrientes (Argentina), con edades entre los 18 y 49 años $(M=21.94, D E=3.39)$, de los cuales el 68.4\% eran mujeres. La escala de atribuciones evidenció una estructura de tres factores: cultural, interna y externa, replicando la propuesta original, aunque con un menor número de ítems. La escala de actitudes mostró una estructura unidimensional; ambas escalas evidenciaron coeficientes alfa de Cronbach superiores a .70. Las relaciones entre las distintas atribuciones y las actitudes emocionales coincidieron parcialmente con lo observado en estudios previos. Se sugieren estudios que permitan obtener evidencia de otras propiedades de las escalas e incorporen componentes no abordados en esta investigación.
\end{abstract}

Palabras clave. Psicometría, pobreza, atribuciones, actitudes, estudiantes universitarios.

Abstract. The aim of this study was to analyze the psychometric properties of the Attributions for Poverty Causes Scale and the Attitudes toward the Poor Scale (affective dimension) in the local context. The sample was composed by 177 college students attending two institutions of Corrientes (Argentina), aged between 18 and 49 years $(M=21.94, S D=3.39)$, $68.4 \%$ women. The first scale showed a three-factor structure: cultural, internal and external, which replicates the original proposal, but with a smaller number of items. The second scale showed an one-dimensional structure. Both scales showed Cronbach alpha coefficients higher than .70. The relationships between the different attributions and emotional attitudes partially overlapped with those observed in previous studies. We suggest new studies in order to obtain evidence for other properties of the scales and incorporate components that are not addressed in this research.

Keywords. Psychometrics, poverty, attributions, attitudes, university students.

\footnotetext{
${ }^{1}$ Cecilia Reyna, Laboratorio de Psicología Cognitiva, Facultad de Psicología, Universidad Nacional de Córdoba; Consejo Nacional de Investigaciones Científicas y Técnicas (CONICET). Dirección postal: Laboratorio de Psicología Cognitiva, Facultad de Psicología, Universidad Nacional de Córdoba, Ciudad Universitaria - CP: 5000, Córdoba, Argentina. E-mail: ceciliareyna@gmail.com

${ }^{2}$ Ma. Florencia Reparaz, Instituto de Investigaciones Científicas, Universidad de la Cuenca del Plata. E-mail: reparazflorencia@hotmail.com
} 


\section{Introducción}

Un trabajo de revisión reciente recuperó investigaciones referidas a las percepciones sobre los pobres y la pobreza desde la década del 70 hasta 2009 (Dakduk, González, \& Malavé, 2010). Dicha revisión pone de manifiesto que la mayoría de los estudios se han realizado en países desarrollados, con escasa proporción de pobres, o con muestras de estudiantes y comunidades que no tienen incidencia directa en las políticas públicas o iniciativas privadas para mejorar la calidad de vida de estas personas. Asimismo, la revisión de Dakduk et al. (2010) evidencia una notable heterogeneidad en cuanto a los constructos abordados y un uso intercambiable de los procesos psicológicos estudiados, por ejemplo, se designan percepciones cuando en realidad se estudian atribuciones o causas. Más allá de eso, éste identificó que gran parte de las investigaciones comprendidas en la revisión abordaban el estudio de atribuciones o causas (44\%) y percepciones de la pobreza $(22 \%)$, mientras que otro grupo de investigaciones comprende a las actitudes como objeto de estudio $(23 \%)$, y el resto una combinación de los constructos mencionados.

En el presente documento, el foco se ubica en el estudio de las atribuciones sobre las causas de la pobreza y las actitudes hacia los pobres por parte de estudiantes universitarios que cursan distintas carreras en la ciudad de Corrientes, una de las ciudades con mayores índices de pobreza económica de Argentina (INDEC, 2012): considerando futuros profesionales que se encontrarán a diario en situaciones que involucren a personas en desventaja económica. Específicamente, se propone analizar de manera preliminar las propiedades psicométricas de dos instrumentos que evalúan atribuciones sobre las causas de la pobreza y actitudes hacia los pobres en el contexto local. A continuación se recuperan conceptualizaciones sobre los constructos de interés y la dimensionalidad observada con muestras de otras latitudes de los instrumentos bajo análisis. Luego, se exponen aspectos metodológicos y se presentan los resultados de cada escala y la relación entre las mismas. Finalmente, se discuten los resultados y se concluye sobre éstos.
Numerosos estudios en Psicología de la Pobreza (Furnham, 2003) abordan la visión de las personas respecto de las causas de la pobreza, lo cual alude al concepto de atribución definido como un proceso a través del cual se intentan comprender las causas de un comportamiento (Baron \& Byrne, 2005). Una clasificación conocida es la propuesta de Feagin (1972), quien identificó una atribución causal tripartita: individualista, las causas se atribuyen a las personas (falta de esfuerzo); estructural, las causas se atribuyen a factores sociales y económicos externos (índices de desempleo); y fatalista, lo cual alude a factores también externos pero asociados, a la mala suerte o circunstancia poco afortunadas. Vale señalar que una misma persona puede atribuir distintas causas, es decir, recurrir a explicaciones mixtas (Palomar Lever \& Pérez Corres, 2003).

Se ha identificado un error fundamental de atribución debido a que si bien se reconocen causas múltiples de la pobreza, las causas individuales han evidenciado ser más importantes que las estructurales (Cozzarelli, Wilkinson \& Tagler, 2001). El estudio de Feagin (1972) y otros posteriores han mostrado que las personas adultas con un nivel de ingresos medios generalmente suscriben a una visión individualista de la pobreza, mientras que las personas de ingresos económicos más bajos es más probable que realicen atribuciones estructurales (Cozzarelli et al., 2001; Hunt 1996).

La valoración de las atribuciones sobre las causas de la pobreza se ha realizado, entre otros, a través de escalas con formato de respuesta cerrada. Uno de tales instrumentos es el desarrollado por Cozzarelli et al. (2001), quienes propusieron la Escala de Atribuciones sobre las Causas de la Pobreza. Dicha escala fue elaborada a partir de ítems de empleados por Feagin (1972) y Smith y Stone (1989) y aplicada a estudiantes universitarios estadounidenses, y ha evidenciado adecuadas propiedades psicométricas en el contexto de su creación. Cozzarelli et al. (2001) identifican tres dimensiones de atribuciones causales, dos semejantes a las comúnmente reconocidas, causas internas y causas externas/sociales, y una referida a la creencia sobre una subcultura de la pobreza, que reemplaza a la atribución fatalista. 
Otra de las variables de interés en esta investigación son las actitudes hacia los pobres, constructo entendido como evaluaciones favorables o desfavorables hacia determinados aspectos del mundo social (Baron \& Byrne, 2005). La importancia de su estudio radica en que las actitudes hacia determinado objeto predicen el comportamiento de las personas hacia dicho objeto, sobre todo cuando son estables, accesibles y están formadas por la experiencia directa (Kraus, 1995).

La literatura sobre actitudes indica que éstas se expresan a través de tres vías: cognitiva, afectiva y conductual; lo cual ha dado lugar a la concepción de elementos o componentes de las actitudes (Morales, 1999). En la presente investigación se recupera el componente afectivo de las actitudes hacia los pobres, expresado como sentimientos evaluativos, preferencias, estados anímicos y emociones que se manifiestan ante el objeto de la actitud. Cozzarelli et al. (2001), elaboraron una escala para medir este componente y la evaluaron con estudiantes universitarios estadounidenses, obteniendo una estructura unidimensional que agrupaba ítems de actitudes favorables y desfavorables (items que fueron invertidos) de contenido afectivo.

Diversos estudios señalan que las atribuciones causales desempeñan un importante papel en la formación de actitudes ante determinados objetos sociales (Hine, Montiel, Cooksey \& Lewko, 2005; Vázquez \& Panadero, 2007). En particular, distintas facetas de las atribuciones sobre las causas de la pobreza se relacionan significativamente con las actitudes hacia los pobres (Cozzarelli et al., 2001; Cozarrelli, Tagler, \& Wilkinson, 2002).

Es importante señalar estudios que, recurriendo a otros constructos teóricos (representaciones sociales), han procurado recuperar la concepción que tienen de la pobreza estudiantes universitarios que ejercerán probablemente sus prácticas de manera cotidiana en contextos como éstos. Denegri et al. (2010) observaron que estudiantes universitarios chilenos de distintas carreras en general, compartían significados comunes en cuanto a pobreza, personas pobres, causas y soluciones. No obstante, los y las estudiantes de la Facultad de Educación y Humanidades le otorgaron mayor importancia a factores de carácter histórico en la existencia de pobres y ricos, mientras que los de la Facultad de Medicina dieron mayor importancia a los factores sociales.

Por último, cabe remarcar las particularidades de la región en la que se encuentran los y las estudiantes universitarios que formaron parte de esta investigación; concretamente, este estudio se desarrolló en la ciudad de Corrientes, Argentina. Según estudios realizados en el año 2003, la provincia de Corrientes era caracterizada por una pobreza estructural que la convertía en la zona de mayor indigencia del país (Rivas, 2003); datos más recientes, también aportan evidencia en esa línea. Según información brindada por el INDEC (2012), el aglomerado de Corrientes sigue presentando las proporciones más elevadas de pobreza (alrededor del $13 \%$ ), de manera semejante a otros aglomerados del Noreste (en especial, Gran Resistencia y Posadas).

De esta manera, resulta de interés contar con herramientas que permitan identificar las atribuciones sobre las causas de la pobreza y las actitudes hacia los pobres por parte de futuros profesionales de esta región. Debido a la ausencia de instrumentos de medición locales, este trabajo se propuso analizar las propiedades psicométricas de dos escalas para medir tales constructos elaboradas por Cozzarelli et al. (2001), específicamente, analizar la estructura factorial, la consistencia interna y la correlación ítem-total, como también la relación entre las distintas atribuciones causales y el componente afectivo de las actitudes hacia los pobres en una muestra de estudiantes universitarios que cursan distintas carreras en la ciudad de Corrientes.

\section{Método}

\section{Participantes}

La muestra estuvo compuesta por 177 estudiantes de distintas carreras (psicología, medicina, nutrición, ciencias económicas, derecho) y años de la Universidad de la Cuenca del Plata y de la Universidad Nacional del Nordeste (Corrientes, Argentina), con edades comprendidas entre los 18 y 49 años $(M=21.94, D E=$ 3.39), siendo el $68.4 \%$ mujeres. El método de muestreo fue no probabilístico, auto-elegido (Sterba \& Foster, 
2008), ya que fue decisión de los y las estudiantes participar o no en el estudio. Los participantes recibieron información de manera oral y escrita sobre los objetivos del estudio y se aseguró el manejo confidencial de la información brindada, empleándose sólo con fines académicos.

\section{Instrumentos}

Attributions for poverty (Cozzarelli et al., 2001). La Escala de Atribuciones sobre las Causas de la Pobreza posee 23 ítems como causas de la pobreza (Tagler, comunicación personal). Ante cada ítem, el o la participante tiene que indicar la importancia de cada uno de ellos como causa de la pobreza sobre una escala tipo Likert de 5 puntos $(1=$ no importante para la pobreza, $5=$ extremadamente importante como causa de pobreza). Los ítems son adaptaciones de los empleados por Feagin (1972) y Smith y Stone (1989). En el estudio de la escala original, el análisis de componentes principales con rotación oblicua evidenció un primer factor que agrupaba a ítems de atribuciones externas focalizadas en oportunidades económicas o financieras y explicó el $21.10 \%$ de la varianza total $(\alpha=.79)$ (p.e., "Prejuicios y discriminación en la contratación de trabajo"), un segundo factor que representaba atribuciones internas y explicó el 14.10\% $(\alpha=.75)$ (p.e., "Falta de esfuerzo y pereza"), y un tercer factor que reflejaba creencias sobre una subcultura de la pobreza más que atribuciones fatalistas como se esperaba, explicando el $7.40 \%$ de la varianza $(\alpha=$ .65) (p.e., "Tener que asistir a escuelas malas"). Los ítems en cada subescala se promedian para brindar una puntuación total donde un puntaje mayor indica que se realizan más atribuciones de ese tipo.

Attitudes toward the poor (Cozzarelli et al., 2001). La Escala de Actitudes hacia los Pobres comprende dos componentes: afectivo y cognitivo, en este estudio se emplearon sólo los 12 ítems de la dimensión afectiva, seis expresados en sentido positivo (como "Me preocupo por la gente pobre") y seis en sentido negativo (como "Trato de evitar relacionarme con la gente pobre"). La valoración de los ítems se realiza sobre una escala tipo Likert de 5 puntos $(1=$ totalmente en desacuerdo, $5=$ totalmente de acuerdo). La consistencia interna medida con el coeficiente alfa de Cronbach fue de .87 en el estudio original con muestras de estudiantes universitarios. Los ítems negativos son invertidos y todos se promedian para brindar una medida total de actitudes positivas hacia los pobres.

\section{Procedimiento}

Para la recolección de datos se solicitó autorización a docentes a cargo de distintas asignaturas y luego se invitó a los ylas estudiantes. La aplicación de los cuestionarios estuvo a cargo de investigadores o estudiantes entrenados, quienes informaron los objetivos del estudio, el carácter voluntario de su participación y la confidencialidad del manejo de los datos; lo cual atañe a los aspectos éticos del estudio (previamente evaluado por la universidad). Estas escalas se aplicaron en conjunto con otros instrumentos que no son objeto de este estudio. Los participantes completaron los cuestionarios individualmente en sus correspondientes grupos de clase, en horario y espacio áulico.

\section{Análisis de datos}

Los datos fueron cargados manualmente en una base de datos y posteriormente analizados, para lo cual se empleó el programa SPSS 20 y AMOS 19. Se realizaron análisis de las propiedades psicométricas y de relaciones entre las variables estudiadas. 1) Se condujo un análisis preliminar de casos y variables: se consideraron casos atípicos univariados aquellos que presentaban valores $Z> \pm 3.29$ y atípicos multivariados a un nivel $p<.001$ (Tabachnick y Fidell, 2001). Valores de asimetría y curtosis en el rango \pm 1 se consideraron excelentes, y en el rango \pm 1.5 fueron aceptables (George y Mallery, 2001). 2) Se analizó la estructura interna de cada escala de manera exploratoria.

Además, se emplearon el método de Componentes Principales (de la misma forma que los autores de la escala original) y el de Ejes Principales. Se evaluó inicialmente la factibilidad del análisis a través del índice de adecuación muestral KMO y el test de esfericidad de Bartlett. Se consideró la regla de Kaiser-Gutman y el gráfico de sedimentación para la interpretación de los factores subyacentes. En estos análisis, se mantuvieron los ítems que presentaban cargas factoriales mayores a .40. 3) Se examinó la consistencia interna a través 
del coeficiente alfa de Cronbach y la correlación ítemtotal corregida. 4) Se analizó la correlación entre la dimensión afectiva de las actitudes hacia los pobres y las distintas dimensiones de las atribuciones sobre las causas de la pobreza.

\section{Resultados}

\section{Escala de Atribuciones sobre las Causas de la Pobreza}

\section{Análisis preliminar}

El análisis inicial evidenció que siete participantes no habían respondido ninguno de los ítems de la escala bajo análisis, por lo cual se optó por excluirlos en análisis posteriores. Además, se observó que ninguna variable presentaba más del 5\% de datos ausentes. Según el test MCAR de Little, la distribución de los mismos no fue completamente aleatoria $\left(\chi^{2}(494)=565.14, p=.014\right)$. Las variables fueron transformadas a dicotómicas para avanzar en la caracterización de los datos ausentes, éstos recibieron el valor de cero y los válidos el valor de uno, y se realizaron correlaciones para observar en qué medida los datos ausentes estaban relacionados entre los pares de variables. Predominaron las correlaciones bajas y no significativas. No obstante, se apreciaron algunas correlaciones moderadas a altas entre algunos pares de ítems $(1$ y $2 r=.40 ; 6$ y $21 r=.71 ; 6$ y $22 r=$ $.71 ; 8$ y $9 r=.70 ; 8$ y $10 r=.40 ; 10$ y $11 r=.51 ; 10$ y $16 r=.40 ; 13$ y $19 r=.49 ; 14$ y $20 r=.57$; en todos

Tabla 1

Estadísticos descriptivos de los items de la Escala de Atribuciones sobre las Causas de la Pobreza

\begin{tabular}{lcccc}
\hline \multicolumn{1}{c}{ Ítems } & $M$ & $D E$ & Asimetría & Curtosis \\
\hline 1. Falta de esfuerzo y pereza. & 3.74 & 1.13 & -0.58 & -0.53 \\
2. No hay intentos de auto-superarse. & 3.71 & 1.14 & -0.61 & -0.37 \\
3. Los ricos se aprovechan de los pobres. & 3.14 & 1.33 & -0.15 & -1.11 \\
4. Enfermedad o discapacidad física. & 3.43 & 1.24 & -0.38 & -0.81 \\
5. Sólo mala suerte. & 1.82 & 1.08 & 1.16 & 0.43 \\
6. Abuso de alcohol y drogas. & 3.71 & 1.25 & -0.70 & -0.51 \\
7. Fracaso de la industria para ofrecer suficientes empleos. & 3.56 & 1.22 & -0.52 & -0.63 \\
8. Prejuicios y discriminación en la contratación de trabajo. & 3.71 & 1.13 & -0.64 & -0.33 \\
9. Falta de habilidad y talento. & 2.63 & 1.14 & 0.23 & -0.71 \\
10. Ausencia de motivación causada por la sensación de bienestar. & 3.22 & 1.20 & -0.21 & -0.76 \\
11. Falta de moral. & 2.82 & 1.22 & 0.08 & -0.85 \\
12. Prejuicios y discriminación en los ascensos y los salarios. & 3.18 & 1.06 & -0.17 & -0.29 \\
13. Haber nacido en la pobreza. & 2.98 & 1.40 & 0.00 & -1.23 \\
14. Haber nacido con un bajo coeficiente intelectual. & 2.71 & 1.24 & 0.29 & -0.75 \\
15. Tener muchos hijos. & 3.11 & 1.36 & -0.06 & -1.12 \\
16. La desintegración de la familia nuclear (padres e hijos). & 2.79 & 1.37 & 0.12 & -1.15 \\
17. Los tipos de trabajos que los pobres pueden obtener son frecuentemente & 3.32 & 1.23 & -0.29 & -0.89 \\
pagados. & & & & \\
18. Tener que asistir a escuelas malas. & 2.84 & 1.42 & 0.15 & -1.30 \\
19. No heredar dinero de parientes. & 1.74 & 1.15 & 1.53 & 1.34 \\
20. Falta de ahorro y manejo apropiado del dinero entre la gente pobre. & 3.29 & 1.32 & -0.37 & -0.97 \\
21. No tener los contactos adecuados que ayuden a encontrar trabajo. & 2.50 & 1.22 & 0.30 & -0.96 \\
22. Un gobierno que es insensible a la difícil situación de la gente pobre. & 3.54 & 1.35 & -0.56 & -0.85 \\
23. Estar desempleado. & 4.15 & 1.16 & -1.40 & 1.12 \\
\hline
\end{tabular}


los casos relaciones significativas a un nivel $p<.001$ ). En función de lo anterior, se optó por reemplazar los valores ausentes con la media, teniendo en cuenta las correlaciones observadas en los análisis posteriores.

Por otra parte, no se apreciaron casos atípicos univariados ni multivariados. Luego, se analizó la distribución de las variables, se observaron valores excelentes de asimetría y curtosis para la mayoría de los ítems, mientras que algunos presentaron valores aceptables de asimetría (ítems 5 y 23) y curtosis (ítems 3 , $15,16,18,19$ y 23), y un ítem mostró un valor de asimetría ligeramente superior a lo aceptable (ítem 19, asimetría = 1.53). Los resultados se pueden observar en la Tabla 1.

\section{Análisis de la estructura factorial}

Como se realizó en el estudio de la escala original, se condujo un Análisis de Componentes Principales con los 23 ítems de la escala, empleándose rotación promax para facilitar la interpretación de los resultados. El índice de adecuación muestral KMO de .729 y el test de esfericidad de Bartlett significativo ( $\chi^{2}$ aprox. (253, $n$ $=170)=1144.01, p<.001)$ avalaron la factibilidad del análisis. La regla de Kaiser-Gutman sugirió la extracción de seis componentes, mientras que el gráfico de sedimentación sugirió la existencia de tres componentes.

En base a lo anterior, se ejecutó un nuevo análisis solicitándose la extracción de tres componentes (Tabla 2). De manera sucesiva, se eliminaron ítems que presentaban cargas factoriales menores a .40 y/o presentaban baja comunalidad (menor a .30) y/o presentaban cargas factoriales complejas, eliminándose en total seis ítems. Los 17 ítems restantes se agruparon en los siguientes componentes: un primer componente agrupó ocho ítems referidos a atribuciones culturales como causas de la pobreza y explicó el $25.02 \%$ de la varianza; un segundo componente agrupó cuatro ítems sobre atribuciones internas y explicó el 13.39\% de la varianza; y un tercer componente agrupó cinco ítems sobre atribuciones externas y explicó el 10.46\% de la varianza (Tabla 2). Posteriormente, los 17 ítems fueron sometidos a un análisis factorial con método de extracción de Ejes Principales (con rotación promax), apreciándose una estructura semejante con sutiles diferencias en las cargas factoriales. El primer factor explicó el $21.50 \%$ de la varianza, el segundo factor el $10.64 \%$, y el tercer factor el $7.14 \%$ (Tabla 2).

\section{Análisis de consistencia interna y correlación item-total}

El examen de la correlación ítem - total corregida muestra valores en el rango .44 a .54 para el factor 1 sobre atribuciones culturales, con un coeficiente alfa de Cronbach de .79; para el factor 2 atribuciones internas los valores de correlación estuvieron en el rango .36 a .69, con un coeficiente alfa de Cronbach igual a .74; y para el factor 3 sobre atribuciones externas las correlaciones estuvieron en el rango de .43 a $.60 \mathrm{y}$ el coeficiente alfa de Cronbach fue de .73 (Tabla 3).

\section{Escala de Actitudes hacia los Pobres}

\section{Análisis preliminar}

$\mathrm{El}$ análisis inicial evidenció que tres participantes no habían respondido los ítems de la escala bajo análisis, por lo cual se optó por excluirlos de los análisis posteriores; además ninguna variable presentó más del $5 \%$ de datos ausentes. Según el test MCAR de Little la distribución de los mismos fue completamente aleatoria $\left(\chi^{2}(210)=197.93, p=.715\right)$. Teniendo eso en cuenta, se optó por reemplazar los datos ausentes empleando los valores de media.

Se observaron seis casos atípicos univariados, en tanto que dos de esos casos resultaron atípicos multivariados. Se optó por excluir los seis casos, por lo cual la muestra para los siguientes análisis quedó compuesta por 168 participantes, posteriormente se analizó la distribución de las variables. Como se aprecia en la Tabla 4, se observaron valores excelentes de asimetría y curtosis para la mayoría de los ítems, y aceptables para dos ítems en cuanto a asimetría (ítems 2 y 7) y para tres ítems en cuanto a curtosis (ítems 5,6 y 10 ).

\section{Análisis de la estructura factorial}

Inicialmente se condujo un Análisis de Componentes Principales, el índice de adecuación muestral KMO de .829 y el test de esfericidad de Bartlett significativo $\left(\chi^{2}\right.$ aprox. $(66, n=168)=556.13, p<.001)$ garantizaron la factibilidad del análisis. La regla de Kaiser-Gutman 
Tabla 2

Saturación factorial de los items de la Escala de Atribuciones sobre las Causas de la Pobreza

\begin{tabular}{|c|c|c|c|c|c|c|c|c|c|}
\hline \multirow{2}{*}{ Ítems } & \multicolumn{3}{|c|}{ ACP1 } & \multicolumn{3}{|c|}{ ACP2 } & \multicolumn{3}{|c|}{$\mathrm{AFE}$} \\
\hline & C1 & $\mathrm{C} 2$ & C3 & C1 & $\mathrm{C} 2$ & $\mathrm{C} 3$ & F1 & $\mathrm{F} 2$ & $\mathrm{~F} 3$ \\
\hline 1. Falta de esfuerzo y pereza. & .01 & -.03 & .86 & -.05 & .90 & -.02 & -.06 & .92 & -.01 \\
\hline 2. No hay intentos de auto-superarse. & -.06 & .02 & .86 & -.08 & .88 & -.03 & -.07 & .84 & -.03 \\
\hline 3. Los ricos se aprovechan de los pobres. & .29 & .25 & -.12 & & & & & & \\
\hline 4. Enfermedad o discapacidad física. & -.18 & .49 & .18 & & & & & & \\
\hline 5. Sólo mala suerte. & .70 & -.18 & -.08 & .71 & -.12 & -.14 & .61 & -.09 & -.11 \\
\hline 6. Abuso de alcohol y drogas. & -.14 & .46 & .40 & & & & & & \\
\hline 7. Fracaso de la industria para ofrecer suficientes empleos. & -.10 & .75 & -.01 & -.03 & -.04 & .71 & -.03 & -.03 & .61 \\
\hline 8. Prejuicios y discriminación en la contratación de trabajo. & -.04 & .81 & -.01 & -.01 & .01 & .81 & -.04 & .01 & .81 \\
\hline 9. Falta de habilidad y talento. & .58 & -.09 & .30 & .63 & .23 & -.06 & .59 & .19 & -.06 \\
\hline 10. Ausencia de motivación causada por la sensación de bienestar. & .29 & .09 & .37 & .22 & .41 & .14 & .22 & .31 & .13 \\
\hline 11. Falta de moral. & -.01 & -.04 & .67 & -.04 & .70 & -.01 & .02 & .53 & -.01 \\
\hline 12. Prejuicios y discriminación en los ascensos y los salarios. & .02 & .56 & .03 & -.08 & .14 & .66 & -.04 & .10 & .54 \\
\hline 13. Haber nacido en la pobreza. & .59 & -.03 & .01 & .61 & -.03 & .01 & .53 & -.02 & .02 \\
\hline 14. Haber nacido con un bajo coeficiente intelectual. & .61 & -.16 & .25 & .58 & .24 & -.10 & .55 & .19 & -.09 \\
\hline 15. Tener muchos hijos. & .50 & .08 & .25 & .48 & .25 & .12 & .44 & .19 & .11 \\
\hline 16. La desintegración de la familia nuclear (padres e hijos). & .45 & .17 & .13 & .45 & .13 & .16 & .39 & .11 & .14 \\
\hline $\begin{array}{l}\text { 17. Los tipos de trabajos que los pobres pueden obtener son } \\
\text { frecuentemente mal pagados. }\end{array}$ & .44 & .35 & -.24 & & & & & & \\
\hline 18. Tener que asistir a escuelas malas. & .46 & .21 & -.01 & & & & & & \\
\hline 19. No heredar dinero de parientes. & .76 & -.12 & -.14 & .77 & -.19 & -.06 & .69 & -.16 & -.05 \\
\hline $\begin{array}{l}\text { 20. Falta de ahorro y manejo apropiado del dinero entre la gente } \\
\text { pobre. }\end{array}$ & .19 & .40 & .24 & & & & & & \\
\hline 21. No tener los contactos adecuados que ayuden a encontrar trabajo. & .66 & .11 & -.14 & .71 & -.21 & .12 & .64 & -.18 & .11 \\
\hline $\begin{array}{l}\text { 22. Un gobierno que es insensible a la difícil situación de la gente } \\
\text { pobre. }\end{array}$ & .08 & .60 & -.27 & .02 & -.20 & .69 & .02 & -.15 & .57 \\
\hline 23. Estar desempleado. & .06 & .56 & .02 & .02 & .07 & .59 & .04 & .06 & .46 \\
\hline
\end{tabular}

Nota. ACP1 $=$ análisis de componentes principales inicial; $\mathrm{ACP} 2=$ análisis de componentes principales final; $\mathrm{AFE}=$ análisis factorial exploratorio con método de extracción de ejes principales; $\mathrm{C}=$ componente $1,2,3 ; \mathrm{F}=$ factor 1, 2, 3 .

sugirió la extracción de tres factores, no obstante, debido a que con dicha regla se tiende a sobreestimar las dimensiones subyacentes (Cohen y Swerdlik, 2010), se interpretó el gráfico de sedimentación, el cual sugirió la existencia de un componente, que presenta un autovalor de 4.28 y explica el $35.63 \%$ de la varianza total. Once de los doce ítems presentaron cargas factoriales superiores a .40 , mientras que el ítem 5 mostró una carga factorial de .39 (Tabla 5).

Luego, se evaluó la estructura factorial empleando un método de extracción factorial, el método de Ejes Principales. Nuevamente, la estructura mostró ser unidimensional, con un único factor que explica el $30.20 \%$ de la varianza tras la extracción. Diez de los 
Tabla 3

Correlación item-total y consistencia interna de la Escala de Atribuciones sobre las Causas de la Pobreza

\begin{tabular}{lcc}
\multicolumn{1}{c}{ Ítems } & rítem-total corregida & $\begin{array}{c}\alpha \text { de Cronbach si el } \\
\text { ítem es eliminado }\end{array}$ \\
\hline Factor 1: Atribuciones culturales $(\alpha=.79)$ & .46 & .77 \\
5. Sólo mala suerte. & .54 & .76 \\
9. Falta de habilidad y talento. & .47 & .77 \\
13. Haber nacido en la pobreza. & .52 & .76 \\
14. Haber nacido con un bajo coeficiente intelectual. & .50 & .76 \\
15. Tener muchos hijos. & .44 & .77 \\
16. La desintegración de la familia nuclear (padres e hijos). & .51 & .76 \\
19. No heredar dinero de parientes. & .51 & .76 \\
21. No tener los contactos adecuados que ayuden a encontrar trabajo. & & \\
Factor 2: Atribuciones internas ( $\alpha=.74)$ & .69 & .59 \\
1. Falta de esfuerzo y pereza. & .65 & .61 \\
2. No hay intentos de auto-superarse. & .36 & .78 \\
10. Ausencia de motivación causada por la sensación de bienestar. & .47 & .72 \\
11. Falta de moral. & & \\
Factor 3: Atribuciones externas $(\alpha=.73)$ & .49 & .68 \\
7. Fracaso de la industria para ofrecer suficientes empleos. & .60 & .64 \\
8. Prejuicios y discriminación en la contratación de trabajo. & .46 & .70 \\
12. Prejuicios y discriminación en los ascensos y los salarios. & .49 & .69 \\
22. Un gobierno que es insensible a la difícil situación de la gente pobre. & .43 & .71 \\
23. Estar desempleado. & & \\
\hline
\end{tabular}

doce ítems mostraron cargas factoriales superiores a .40 y dos ítems superiores a .30 (ítems 5 y 12) (Tabla 5).

\section{Análisis de correlación item-total y consistencia interna}

Se observaron valores de correlación ítem-total corregida entre .31 y .63. Los valores más bajos los presentaron los ítems 5 y 12. La consistencia interna evaluada con el coeficiente alfa de Cronbach resultó aceptable con un valor de .83. Los resultados se detallan en la Tabla 5.

Relaciones entre Actitudes hacia los Pobres y Atribuciones sobre las Causas de la Pobreza

Las atribuciones culturales se relacionaron positiva y significativamente con las atribuciones internas $(r=.31$, $p<.001)$ y externas $(r=.29, p<.001)$, mientras que las atribuciones internas y externas no se relacionaban de manera significativa. En cuanto a las relaciones entre las actitudes emocionales hacia los pobres, se observaron relaciones positivas con atribuciones externas $(r=.23$, $\mathrm{p}=.003)$ y negativas con atribuciones culturales $(r=$ $-.19, p=.015)$ e internas $(r=-.27, p<.001)$, es decir que quienes realizan más atribuciones externas presentan actitudes más positivas hacia los pobres, y quienes hacen más atribuciones culturales o internas muestran actitudes menos favorables hacia los pobres.

\section{Discusión}

El objetivo de este estudio fue evaluar las propiedades psicométricas de la Escala de Atribuciones sobre las Causas de la Pobreza, y la dimensión afectiva de la Escala de Actitudes hacia los Pobres propuestas por 
Tabla 4

Estadísticos descriptivos de los items de la Escala de Actitudes hacia los Pobres

\begin{tabular}{lcccc}
\hline \multicolumn{1}{c}{ Ítems } & $M$ & $D E$ & Asimetría & Curtosis \\
\hline 1.r La gente pobre me hace sentir incómodo. & 3.98 & 0.93 & -0.75 & 0.39 \\
2.r No me agradan mucho las personas pobres. & 4.37 & 0.78 & -1.13 & 0.75 \\
3. Mis sentimientos hacia las personas pobres son generalmente positivos. & 3.40 & 0.84 & -0.22 & 0.48 \\
4.r Trato de evitar relacionarme con la gente pobre. & 4.07 & 0.87 & -0.52 & -0.64 \\
5. Tengo gran estima por la gente pobre. & 3.17 & 0.82 & -0.20 & 1.08 \\
6. Me preocupo por la gente pobre. & 3.70 & 0.82 & -0.77 & 1.47 \\
7. Siento que las personas pobres son dignas de respeto. & 4.32 & 0.87 & -1.12 & 0.37 \\
8.r Tengo sentimientos negativos hacia las personas pobres. & 4.31 & 0.79 & -1.00 & 0.47 \\
9.r Tengo una mala opinión de las personas pobres. & 4.11 & 0.90 & -0.81 & 0.12 \\
10. Generalmente, la gente pobre me cae bien. & 3.23 & 0.74 & 0.04 & 1.54 \\
11.r A menudo reacciono negativamente hacia la gente pobre. & 3.93 & 0.92 & -0.84 & 0.69 \\
12. No tengo dudas de interactuar con la gente pobre. & 3.69 & 0.93 & -0.56 & 0.31 \\
\hline
\end{tabular}

Nota. La puntuación de los ítems con $r$ fue invertida.

Tabla 5

Saturación factorial de los items de la Escala de Actitudes hacia los Pobres

\begin{tabular}{lcccc}
\hline \multicolumn{1}{c}{ Ítems } & ACP & AFE & $\begin{array}{c}r \text { ítem-total } \\
\text { corregida }\end{array}$ & $\begin{array}{c}\alpha \text { de Cronbach si el } \\
\text { ítem es eliminado }\end{array}$ \\
\hline 1.r La gente pobre me hace sentir incómodo. & .59 & .54 & .47 & .82 \\
2.r No me agradan mucho las personas pobres. & .74 & .71 & .63 & .80 \\
3. Mis sentimientos hacia las personas pobres son generalmente positivos. & .61 & .55 & .51 & .81 \\
4.r Trato de evitar relacionarme con la gente pobre. & .72 & .68 & .60 & .81 \\
5. Tengo gran estima por la gente pobre. & .39 & .34 & .31 & .83 \\
6. Me preocupo por la gente pobre. & .54 & .47 & .44 & .82 \\
7. Siento que las personas pobres son dignas de respeto. & .56 & .50 & .46 & .82 \\
8.r Tengo sentimientos negativos hacia las personas pobres. & .68 & .64 & .57 & .81 \\
9.r Tengo una mala opinión de las personas pobres. & .67 & .63 & .57 & .81 \\
10. Generalmente, la gente pobre me cae bien. & .48 & .42 & .40 & .82 \\
11.r A menudo reacciono negativamente hacia la gente pobre. & .64 & .59 & .52 & .81 \\
12. No tengo dudas de interactuar con la gente pobre. & .42 & .37 & .34 & .83 \\
\hline
\end{tabular}

Nota. ACP = análisis de componentes principales; AFE = análisis factorial exploratorio con método de extracción de ejes principales. 
Cozzarelli et al. (2001) en el contexto local, empleando muestras de estudiantes universitarios que asisten a instituciones educativas de Corrientes.

Para cada una de las escalas se analizó la estructura factorial, la consistencia interna y la correlación ítemtotal, y también se examinaron las relaciones entre las distintas atribuciones causales y el componente afectivo de las actitudes hacia los pobres. En términos generales, se apreciaron buenas propiedades psicométricas para ambos instrumentos, aunque en el caso de la Escala de Atribuciones sobre las Causas de la Pobreza algunos ítems no fueron retenidos en la estructura final.

En el análisis inicial de la estructura factorial de la Escala de Atribuciones sobre las Casusas de la Pobreza si bien indicó que era factible proceder dicho análisis teniendo en cuenta la correlación entre los ítems, arrojó una estructura de difícil interpretación, sumado a que varios ítems presentaban carga factorial o comunalidad baja, o presentaban cargas complejas (cargaban alto en más de un factor). Debido a ello, la escala se redujo a 17 ítems, que se agruparon en tres factores sobre atribuciones causales de la pobreza: causas culturales, causas internas o individuales, y causas externas o sociales. Esta estructura replica lo observado por Cozzarelli et al. (2001) en su estudio con estudiantes universitarios estadounidenses, surgiendo una dimensión referida a una subcultura de la pobreza en vez de la dimensión fatalista comúnmente reportada en la literatura. No obstante, a diferencia del estudio de Cozzarelli et al. (2001), en el presente estudio varios ítems resultaron problemáticos y fueron descartados.

La inspección de la estructura factorial de la dimensión afectiva de la Escala de Actitudes hacia los Pobres evidenció una estructura unidimensional, tanto empleando el método de componentes principales como un análisis factorial con método de extracción de ejes principales; en ambos casos la proporción de varianza explicada fue superior al 30\%. Las cargas factoriales fueron, en general, adecuadas. Sólo los ítems 5 ("Tengo gran estima por la gente pobre") y 12 ("No tengo dudas de interactuar con la gente pobre") mostraron cargas inferiores a .40 pero superiores a $.30 \mathrm{en}$ el análisis factorial, por lo cual se decidió conservarlos.
Ambas escalas evidenciaron buenas propiedades de consistencia interna, con coeficientes alfa de Cronbach en el rango de .73 a .79 para la Escala de Atribuciones sobre las Causas de la Pobreza y de .83 para la Escala de Actitudes hacia los Pobres. También resultaron adecuados los valores de correlación ítem-total para cada una de las dimensiones de la primera escala y de la dimensión afectiva evaluada de la segunda escala.

Con respecto a las relaciones entre las atribuciones causales y las actitudes emocionales hacia los pobres, los resultados coinciden parcialmente con lo reportado por Cozzarelli et al. (2001). Quienes realizan atribuciones causales externas de la pobreza manifiestan actitudes emocionales más positivas hacia los pobres, mientras que quienes realizan atribuciones internas evidencian actitudes más negativas. En cambio, a diferencia de lo observado por Cozzarelli et al. (2001), niveles más altos de atribuciones culturales se relacionaron negativamente con la dimensión afectiva de las actitudes hacia los pobres, lo cual evidencia la particularidad de cada contexto.

Si bien para ambos instrumentos se obtuvo evidencia de adecuadas propiedades psicométricas, vale señalar algunas limitaciones de esta investigación que podrían ser tomadas en cuenta en estudios futuros. Por una parte, el tipo de muestreo utilizado fue no probabilístico, por lo que la generalización de los resultados es limitada; además, sería positivo contar con estudios que incorporen otras muestras. Además, se remarca el carácter preliminar de los estudios psicométricos de las escalas analizadas. Se recomienda avanzar en la obtención de otros tipos de evidencia, como por ejemplo, validez convergente/ discriminante y validez de criterio. También se sugiere avanzar en el análisis de otras dimensiones de las actitudes hacia los pobres (cognitivo y comportamental) e incorporar características particulares de los pobres dado que no son un grupo homogéneo, identificando así, por ejemplo, actitudes hacia las mujeres pobres tal como lo han hecho investigaciones previas (Cozzarelli et al., 2002).

De particular relevancia es recuperar la perspectiva de las personas que viven en condiciones de pobreza para ser más precisos en la descripción y análisis acerca de cómo las personas experimentan estas condiciones, 
lo cual resulta crucial como punto de partida para futuros trabajos; como señala Mtapuri (2008), como una forma de comprender su situación y encontrar puntos de entrada para favorecer los canales de salida de la pobreza. Es importante indicar que esta no es una sugerencia original; El Banco Mundial publicó hace poco más de 10 años un estudio que implicó comprender cómo la gente que vive en condiciones de pobreza comprende y define la pobreza (Narayan, 2001), reconociéndose que la pobreza es específica a las locaciones, y aún más, a grupos sociales, aunque existen aspectos comunes de la experiencia humana de la pobreza que atraviesa las fronteras de los países. Así, estudios futuros podrían incorporar $\mathrm{y} / \mathrm{o}$ focalizarse en personas que viven en las condiciones señaladas, para estudiar las propiedades psicométricas de las escalas de atribuciones y actitudes hacia la pobreza.

\section{Conclusiones}

Los estudios psicométricos de la Escala de Atribuciones sobre las Causas de la Pobreza y de la dimensión afectiva de la Escala de Actitudes hacia los Pobres evidenciaron buenas propiedades, tanto en cuanto a la estructura subyacente como a la consistencia interna de las mismas. Estos resultados avalan su uso en el contexto correntino en el marco de investigación, requiriéndose futuros estudios para que sea factible extender su utilización.

\section{Referencias}

Baron, R. A., \& Byrne, D. (2005). Psicología Social (10a ed.). Madrid: Pearson Educación.

Cohen, R. J., y Swerdlik, M. E. (2010). Psychological testing and assessment: $A n$ introduction to tests and measurement (7th ed.). United States of America: McGraw-Hill.

Cozzarelli, C., Tagler, M. J., \& Wilkinson, A. (2002). Do middle-class students perceive poor women and poor men differently? Sex Roles, 47(11/12), 519-529.

Cozzarelli, C., Wilkinson, A., \& Tagler, M. (2001). Attitudes toward the poor and attributions for poverty. Journal of Social Issues, 57(2), 207-227.
Dakduk, S., González, M., \& Malavé, J. (2010). Percepciones acerca de los pobres y la pobreza: una revisión. Revista Latinoamericana de Psicología, 43(3), 413-425.

Denegri, M., Cabezas, D., Sepúlveda, J., del Valle, C., González, Y., \& Miranda,H. (2010). Representaciones sociales sobre pobreza en estudiantes universitarios chilenos. Liberabit, 16(2), 161-170.

Feagin, J. (1972). Poverty we still believe that God helps who help themselves. Psychology Today, 6, 101-129.

Furnham, A. (2003). Belief in a just world: research progress over the past decade. Personality and Individual Differences, 34, 795-817.

George, D., y Mallery, M. P. (2001). SPSS for Windows step by step: A simple guide and reference. Boston, MA: Allyn\& Bacon.

Hine, D. W., Montiel, C. J., Cooksey, R. W., \& Lewko, J. H. (2005). Mental models of poverty in developing nations: A causal mapping analysis using a CanadaPhilippines contrast. Journal of Cross-CulturalPsychology, 36, 283-303.

Hunt, M. (1996). The individual, society, or both? A comparison of Black, Latino and White beliefs about causes of poverty. Social Forces, 75(1), 293-322.

INDEC (2012). Encuesta permanente de hogares. Incidencia de la pobreza y de la indigencia. Resultados del segundo semestre 2011. Recuperado de http://www.indec.gov.ar/ nuevaweb/cuadros/74/pob_tot_2sem11.pdf

Kraus, S. J. (1995). Attitudes and the prediction of behavior: A meta-analysis of the empirical literature. Personality and Social Psychology Bulletin, 21, 58-75.

Morales, J. F. (Coord.) (1999). Psicología Social (2a ed.). Madrid: McGraw-Hill.

Mtapuri, O. (2008). Exploring local conceptions of poverty wealth and well-being: field evidence from Mashonaland West Province of Zimbabwe. Africa Development, XXXIII(3), 35-54.

Narayan, D., 2001, 'Voices of the Poor', in B. Deryke, R. Calderisi and C. Sigden, eds., Faith in Development, 
World Bank and Regnum Books International, (pp. 3948). Oxford.

Palomar Lever, J., \& Pérez Corres, A. (2003). Un solo rostro y tres maneras de mirarlo: el significado de "pobreza" según el nivel socioeconómico. Revista Latinoamericana de Psicología, 35(1), 27-39.

Rivas, A. (2003). Proyecto "Las provincias educativas: Estudio comparado sobre el Estado, el poder y la educación en las 24 jurisdicciones argentinas: Provincia de Corrientes (versión definitiva)". Recuperado de http://cippec. org/proyectoprovincias/

Smith, B., \& Stone, L. H. (1989). Rags, riches, and bootstraps: Beliefs about the causes of wealth and poverty. Sociological Quarterly, 30, 93-107.
Sterba, S. K., \& Foster, M. (2008). Self-selected sample. In P. J. Lavrakas (Ed.), Encyclopedia of Survey Research Methods (pp. 806-808). California: SAGE Publications.

Tabachnick, B., \& Fidell, L. (2001). Using multivariate statistics (4 ed.). Nueva York: Harper \& Row.

Vázquez, J. J., \& Panadero, S. (2007). Ideología, acción política y atribuciones causales de la pobreza en los estados menos desarrollados. Psicología Política, 35, 3551.

Vázquez, J. J., \& Panadero, S. (2009). Atribuciones causales de la pobreza en los países menos desarrollados. Perfiles Latinoamericanos, 34, 125-140.

Recibido: 10 de octubre de 2013

Aceptado: 13 de febrero de 2014 